\title{
Interférences
}

Ars scribendi

$9 \mid 2016$

Varia

\section{Ingénuité et subtilité de Servius}

\section{Giampiero Scafoglio}

\section{(2) OpenEdition}

Journals

Édition électronique

URL : http://journals.openedition.org/interferences/5741

DOI : 10.4000/interferences.5741

ISSN : $1777-5485$

Éditeur

HiSoMA - Histoire et sources des Mondes antiques

Référence électronique

Giampiero Scafoglio, «Ingénuité et subtilité de Servius », Interférences [En ligne], 9 | 2016, mis en ligne le 18 janvier 2018, consulté le 15 septembre 2020. URL : http://journals.openedition.org/interferences/ 5741 ; DOI : https://doi.org/10.4000/interferences.5741

Ce document a été généré automatiquement le 15 septembre 2020.

Tous droits réservés 


\title{
Ingénuité et subtilité de Servius
}

\author{
Giampiero Scafoglio
}

1 Tout le monde connaît la 'naïveté', l'ingénuité de Servius, sa tendance à la simplification et à la banalisation dans l'interprétation des contenus conceptuels ainsi que de la langue virgilienne ${ }^{1}$. Voici quelques exemples tirés du commentaire sur le livre 7 de l'Énéide.

2 Turnus, après avoir rencontré en rêve Allecto, qui a enflammé son cœur par une torche flamboyante, se réveille dans les affres d'un désir effréné de guerre $(A .7,460)$ :

arma amens fremit, arma toro tectisque requirit.

3 L'expression arma... fremit ne peut pas être traduite littéralement ${ }^{2}$ : la construction transitive du verbe fremo est très incisive et efficace ; Virgile l'utilise pour donner l'idée de la recherche effrénée des armes ${ }^{3}$. Servius explique :

FREMIT cum clamore deposcit. bene autem quia in toro requirebat.

4 L'érudit ne fait que rendre explicite le sens de la phrase, qui cependant peut être facilement deviné : il reformule la sentence par une paraphrase plus régulière et linéaire, tandis qu'il n'accorde pas la moindre attention à la construction exceptionnelle et audacieuse de l'expression. Il semble que son seul but soit d'expliquer la signification de la poésie, de la faire clairement comprendre, et non d'en analyser le style ni d'en identifier les expédients expressifs, ni même d'en évaluer les résultats. Le Seruius auctus ajoute :

FREMIT cum fremitu et clamore deposcit. bene autem amens quia in toro requirebat.

L'érudit ressent le besoin d'ajouter le terme fremitu, qui a la même racine que le verbe fremo, en faisant une tautologie, une répétition inutile.

5 Il est clair que Servius (ainsi que l'autre savant qui a élargi son lemme) n'a aucun intérêt à étudier et expliquer une structure linguistique particulière; il préfère certainement la ramener à une formulation ordinaire, qui s'accorde avec son but didactique (en supposant que son commentaire soit vraiment lié à l'enseignement scolaire $)^{4}$. Donc, Servius se révèle ici plutôt superficiel, ou bien il joue simplement son rôle de grammaticus. 
6 D'autre part, l'ingénuité de Servius n'est pas toujours explicable en tant que superficialité ou sur la base de ses objectifs éducatifs. Parfois, il donne des explications complexes et approfondies, mais qui ne sont pas pertinentes, ne sont pas cohérentes avec le contenu du poème.

7 Par exemple, quand Ascagne lance la flèche qui tue le cerf élevé par les garçons et la fille de Tirrus (le berger du roi Latinus), Virgile dit : nec dextrae erranti deus afuit (A. 7, 498) ${ }^{5}$. Servius explique :

DEVS AFVIT Allecto deum dicit, sicut de Venere 'descendo ac ducente deo' <2, 632> : nam ut diximus, numina utriusque sexus uidentur ideo, quia incorporea sunt et quod uolunt adsumunt. ut autem deum dicamus pro dea non aperte ponitur, sed in subauditione.

8 Servius explique que "les dieux possèdent les deux sexes (masculin et féminin), puisqu'ils sont incorporels et peuvent prendre l'apparence qu'ils veulent ${ }^{6} »$. L'utilisation du masculin se référant à une déesse, toutefois, non aperte ponitur, sed in subauditione, "n'est pas pratiquée ouvertement, mais implicitement ». Servius exprime une conception abstraite de la divinité, une idée très raffinée ou, si l'on préfère, philosophique : c'est pourquoi il dit non aperte ponitur, car ce n'est pas un concept d'usage courant. Ici on ne peut pas considérer que Servius s'avère naïf ; au contraire, il propose une explication sophistiquée, qui révèle une ouverture d'esprit remarquable et même un haut niveau de conscience théologique. Toutefois, cette interprétation ne semble pas congruente à l'expression virgilienne, qui se réfère génériquement au succès du lancement de la flèche ou, tout au plus, à la faveur divine qui accompagne le lancement et le rend efficace. Il est peu probable que ce deus, le dieu abstrait qui aide Ascagne, puisse être identifié avec Allecto, pour laquelle Virgile aurait utilisé plus probablement le mot féminin dea (comme il le fait, par ex., en A. 7, 511, saeua... dea). Alors, l'ingénuité de Servius ne consiste pas ici dans une explication simpliste ou triviale, mais dans la mauvaise utilisation d'une théorie sophistiquée.

9 Cependant, l'ingénuité de Servius est connue de tous: la liste des exemples qui l'illustrent pourrait s'étendre longuement. Ce qui en réalité est inconnu ou, du moins, pas suffisamment reconnu, c'est la finesse de Servius : sa subtilité, sa capacité à saisir et à expliquer des aspects structurels et esthétiques qui ne sont ni simples ni triviaux.

10 Voici quelques exemples tirés du commentaire sur le livre 7 de l'Énéide. Ce livre s'ouvre avec la notification (sous la forme d'une apostrophe) de la mort de Caieta, la nourrice d'Enée $(A .7,1-4)^{7}$ :

Tu quoque litoribus nostris, Aeneia nutrix, aeternam moriens famam, Caieta, dedisti ; et nunc seruat honos sedem tuus, ossaque nomen Hesperia in magna, si qua est ea gloria, signat.

11 Ce morceau est commenté dans son ensemble par Servius dans sa scholie au vers 4, juste après deux observations précises et limitées, respectivement sur les expressions Hesperia in magna et si qua est ea gloria. Servius dit alors :

Bene autem interest funeri postquam ab inferis rediit, sicut interfuit antequam descenderet, ut medium actum ostenderet.

12 Même si la formulation est très concise et elliptique, le concept est clair : Virgile a placé de manière appropriée des funérailles avant et après la descente d'Énée aux enfers, à savoir les funérailles de Misène avant la descente du héros $(A .6,162-235)$ et celles de Caieta après son retour au monde des vivants. Servius note donc que la descente d'Énée aux enfers est encadrée, pour ainsi dire, par deux morts avec leurs rites funéraires; 
l'érudit ajoute également une évaluation esthétique (bene... interest...), en soulignant la pertinence des funérailles par rapport au récit qu'elles entourent, récit qui se déroule précisément dans le royaume des morts.

Or il est évident que la mort de Misène remplit une fonction d'introduction, de préparation, par rapport à la descente d'Énée aux enfers : en effet, une telle entreprise n'est possible qu'après l'accomplissement des rites funéraires pour le jeune homme mort, comme la Sibylle elle-même le dit à Énée (A. 6, 149-153). En revanche, la fonction structurale (à la manière d'une conclusion) donnée par le poète à la mort de Caieta par rapport à la descente aux enfers n'est pas si évidente: cette fonction est habilement déguisée grâce à la collocation de l'événement au début du livre suivant. Virgile évite ainsi d'enfermer la descente aux enfers dans un décor trop rigide : le déplacement de la mort de Caieta au début du livre suivant vise le double objectif de rendre le récit plus fluide et plus souple, et d'augmenter la cohésion entre les livres 6 et $7^{8}$.

Le cadre structurel formé par les deux épisodes de funérailles, celles de Misène et celles de Caieta, n'est donc pas immédiatement évident; toutefois il est rapporté (de façon concise, mais claire et précise) par Servius, qui ici ne semble pas du tout naif, mais s'avère un interprète aigu et raffiné en ce qui concerne la construction et la stratégie de la narration.

Un autre exemple intéressant, tiré du commentaire sur le livre 7 mais qui concerne un aspect purement esthétique, porte sur la description évocatrice de la mer nocturne sur laquelle voguent les navires troyens (A. 7, 8-9):

aspirant aurae in noctem nec candida cursus

luna negat, splendet tremulo sub lumine pontus.

Servius explique l'expression tremulo sub lumine en disant: ita enim mobilitas aquae facit uideri. La mer brille sous le clair de lune qui se reflète sur l'eau: c'est une lumière vacillante à cause du mouvement des vagues. Servius comprend et explique de manière adéquate cette image élégante et suggestive, qui vient d'Apollonios de Rhodes et exprime la sensibilité typiquement hellénistique concernant les effets de lumière et de couleur'.

Un cas semblable, mais encore plus marqué, se trouve dans le commentaire sur le livre 8 , notamment dans la description de la navigation sur le Tibre $(A .8,94-96)$ :

olli remigio noctemque diemque fatigant

et longos superant flexus, uariisque teguntur

arboribus, uiridisque secant placido aequore siluas.

L'expression uiridisque secant placido aequore siluas peut être interprétée comme signifiant simplement que " les navires vont à travers les bois », car ils naviguent sur la rivière qui coule au milieu de la végétation ${ }^{10}$. Servius, cependant, préfère une explication plus subtile et sophistiquée, en disant :

SECANT PLACIDO AEQUORE SILVAS ostendit adeo perspicuam fuisse naturam fluminis, ut in eo apparerent imagines nemorum, quas Troianae naues secabant.

19 Selon Servius, dans la description virgilienne les navires troyens « fendent » les images des forêts qui se reflètent dans les eaux du Tibre. Je ne sais pas si c'est là véritablement l'interprétation correcte; mais c'est certainement une interprétation raffinée, tout autre que simple ${ }^{11}$. En effet, il me semble qu'ici Virgile se contente de dire que les navires passent au milieu des bois, qui sont situés sur les rives du fleuve. L'utilisation du verbe seco est compatible avec les deux interprétations, comme le montrent deux passages du même livre. L'interprétation la plus simple et la plus évidente est 
confirmée par la description du Tibre « qui frôle les rives et coule à travers les champs fertiles » $($ A. 8, 63) :

stringentem ripas et pinguia culta secantem.

En revanche, l'interprétation de Servius semble confirmée par la belle description des dauphins qui "frappaient la surface de la mer avec leur queue et fendaient les eaux » (A. 8, 673-674):

et circum argento clari delphines in orbem aequora uerrebant caudis aestumque secabant.

Quelle que soit l'interprétation correcte, il n'y a aucun doute que Servius agit comme un lecteur avisé et perspicace, peut-être trop subtil.

21 En conclusion, Servius n'est pas toujours un commentateur naïf, ingénu, ce qui n'est qu'un euphémisme pour dire stupide. Dans son commentaire il y a :

- des observations naïves;

- des observations sophistiquées, mais incompatibles avec le texte de Virgile ;

- des observations raffinées ainsi que plausibles.

Cela dépend probablement des différentes sources d'où il tire parfois ses observations. Dans certains cas, on ne peut exclure sa contribution personnelle, notamment dans l'évaluation des descriptions naturalistes, telles que les images qui se reflètent dans les eaux. Ici, Servius révèle une sensibilité particulière pour la nature et ses spectacles, la sensibilité visuelle et esthétique typique de l'Antiquité tardive, comme on le voit également, par exemple, dans la Moselle d'Ausone ${ }^{12}$.

Cela confirme l'extraordinaire richesse du commentaire de Servius qui, malgré ses limites, parvient toujours à donner des aperçus intéressants et même à surprendre le lecteur.

\section{BIBLIOGRAPHIE}

\section{Textes anciens}

Virgil, Aeneid. Book VIII, ed. K. W. GRANSDEN, Cambridge Greek and Latin Classics, Cambridge, Cambridge University Press, 1976.

P. Vergili Maronis, Aeneidos. Libri VII-VIII, with a commentary by C. J. FORDYCE ; introd. by P. G. WALSH ; ed. by J. D. CHRISTIE, Glasgow University Publications, Oxford, Oxford University Press, 1977.

Servio, Commento al libro VII dell'Eneide di Virgilio. Con le aggiunte del cosiddetto Servio Danielino, introd., bibliografia, ed. critica a cura di G. RAMIRES, Testi e manuali per l'insegnamento universitario del latino 78, Bologne, Pàtron Ed., 2003. 


\section{Études modernes}

BARCHIESI A. 1979, « Palinuro e Caieta: Due 'epigrammi’ virgiliani (Aen. V.870 sg.; VII.1-4) », Maia 31, p. 3-11.

CASALI S. 2009, "The Theophany of Apollo in Virgil Aeneid 9: Augustanism and Self-Reflexivity », in L. ATHANASSAKI, R. P. MARTIN, J. F. Miller (ed.), Apolline Politics and Poetics, Athènes, Hellenic Ministry of Culture - European Cultural Centre of Delphi, p. 299-327.

EDEN P. T. 1975, A Commentary on Virgil. Aeneid VIII, Mnemosyne. Suppl. 35, Leyde, Brill. ERNOUT A., MEILLET A. 1985, Dictionnaire étymologique de la langue latine, $4^{\mathrm{e}}$ éd. par J. ANDRÉ, Paris, Klincksieck. FERNANDELli M. 2007, « Ombre sull'acqua, da Virgilio a Pascoli », CentoPagine 1, p. 95-102. HARRISON E. L. 1976, « Structure and Meaning in Vergil's Aeneid », Papers of the Liverpool/Leeds/ Langford Latin Seminar 1, p. 101-112.

HORSFALL N. 2000, Virgil, Aeneid 7. A Commentary, Mnemosyne. Suppl. 198, Leyde, Brill. JEUNET-MANCY E. 2007, «Le Commentaire de Servius à l'Énéide : éclectisme ou encyclopédisme? ", Schedae 2, p. 15-25 [en ligne]. URL : https://www.unicaen.fr/puc/images/preprint0022007.pdf. KASTER R. 1988, Guardians of Language. The Grammarian and Society in Late Antiquity, The Transformation of the Classical Heritage 11, Berkeley, University of California Press.

LEVY H. L. 1971, « Servius in his classroom », CJ 67, p. 167-174.

NELIS D. 2001, Vergil's Aeneid and the Argonautica of Apollonius Rhodius, Arca 39, Leeds, F. Cairns. SCAFOGLIO G. 2001, « Tecnica allusiva ed aemulatio nella Mosella di Ausonio », in U. CRISCUolo (ed.), MNEMOSYNON. Studi di letteratura e di umanità in memoria di Donato Gagliardi, Pubblicazioni del Dipartimento di Filologia classica F. Arnaldi 19, Naples, La buona stampa, p. 447-462.

- 2005, « La descrizione della natura nell'Eneide. Osservazioni teoriche e prove di analisi », Euphrosyne 33, p. 355-364.

THOMAS R. 1999, Reading Virgil and His Texts. Studies in Intertextuality, Ann Arbor, The University of Michigan Press.

UHL A. 1998, Servius als Sprachlehrer. zur Sprachrichtigkeit in der exegetischen Praxis des spätantiken Grammatikunterrichts, Hypomnemata 117, Göttingen, Vandenhoeck und Ruprecht.

\section{NOTES}

1. Cf. par ex. KASTER 1988, p. 189 : «Servius's insistent and complacent didacticism makes his observations unreliable and sometimes bizarre, but not disingenuous ». UHL 1998, passim, soutient que l'exégèse de Servius est souvent étonnante, répétitive, maladroite, et que, pour porter un jugement correct sur son commentaire, il faut le lire du point de vue d'un élève, à qui il s'adresse en priorité.

2. En effet, la signification étymologique du verbe fremo est " gronder » et il « se dit de tout bruit grave et violent », comme l'expliquent ERNOUT, MEILLET 1985, p. 252-253. 
3. Cf. Accius, Trag. 588 = Epig., frg. 2 Dangel: Argiuos fremere bellum; également A. 11, 453 : fremit arma iuuentus.

4. Voir par ex. LEVY 1971, p. 167-174; KASTER 1988, p. 169-197, notamment p. 170 : le commentaire de Servius est "the instrument of a teacher » puisqu'il "remains at a lever suitable for pueri as Servius makes his way word by word and line by line through the text »; JEUNET-MANCY 2007, p. 15-26, notamment p. 17 : «Servius joue à plein son rôle de magister et son commentaire s'apparente alors essentiellement à un manuel scolaire ».

5. Qui est le deus dont on parle? «Allecto or not? Discussion continues [...] not always helpfully » (HORSFALL 2000, ad loc.). Voir l'interprétation très suggestive de CASALI 2009, notamment p. 308.

6. Il dit la même chose en $A .2,632$, à propos de l'expression tout aussi énigmatique ducente deo: secundum eos qui dicunt utriusque sexus participationem habere numina; à l'appui de cette explication, il évoque même "une statue de Vénus barbue " qui se trouve dans l'île de Chypre.

7. Sur ce passage, qui semble presque une 'épitaphe' insérée dans l'Énéide, voir FORDYCE 1977, p. 51-52 ; BARCHIESI 1979, p. 3-11 ; THOMAS 1999, p. 101-113 ; HORSFALL 2000, p. 46.

8. D'autant plus que la ville de Gaeta, qui porte le nom de la nourrice d'Énée, est déjà mentionnée à la fin du livre 6, notamment v. 900-901: Tum se ad Caietae recto fert litore portum. / ancora de prora iacitur; stant litore puppes. Voir HARRISON 1976, notamment p. 103.

9. Cf. Apollonios de Rhodes, 1, 594 et 1, 600-601, mais également Ennius, frg. 122, 250 (un modèle qui est déjà rapporté par Macrobe, 6, 4, 7) ; NELIS 2001, p. 257.

10. C'est l'interprétation de la plupart des chercheurs depuis la seconde moitié du $x^{e}$ s. : voir par ex. EDEN 1975, p. 53 : «they row between small islets of clumps and trees, and 'cut through them' »; GRANSDEN 1976, p. 95 : «they make way on the smooth surface between green trees $»$.

11. Cette interprétation, partagée dans le passé par plusieurs chercheurs (tels que Conington, Peerlkamp, Forbiger, Sabbadini, Mackail, ainsi que le poète Giovanni Pascoli), est encore défendue par SCAFOGLIO 2005 et par FERNANDELLI 2007.

12. Cf. Ausone, Mos. 188-199 et 222-229 ; mais aussi Pétrone, Ciu. 119, 28 ; Pline le Jeune, Ep. 8, 8, 4 ; Stace, Silu. 1, 3, 17-19 et 2, 2, 48-49. Sur le développement diachronique de ce thème littéraire, voir SCAFOGLIO 2001.

\section{RÉSUMÉS}

Cet article vise à démontrer, à travers quelques exemples tirés du commentaire sur les livres 7 et 8 de l'Énéide, que Servius n'est pas toujours un commentateur naiff, ingénu (ce qui n'est qu'un euphémisme pour dire stupide). Au contraire, il s'avère parfois un interprète aigu et raffiné en ce qui concerne la construction et la stratégie de la narration virgilienne, tout comme il exprime 
une sensibilité particulière pour la nature et ses spectacles, la sensibilité visuelle et esthétique typique de l'Antiquité tardive.

This paper aims to demonstrate, by means of some examples drawn from the commentary on books VII and VIII of the Aeneid, that Servius is not always a naïve scholar (an euphemism to say stupid). On the contrary, sometimes he proves to be a keen and sophisticated scholar as for the interpretation of the structure and narrative strategy in Virgil's tale, as well as he reveals a special sensitivity to nature and its spectacles: the aesthetic and visual sensitivity which is typical of Late Antiquity.

INDEX

nomsmotscles Apollonios de Rhodes, Ausone, Ennius, Macrobe, Pétrone, Pline le Jeune, Servius Honoratus (Maurus), Stace, Virgile

\section{AUTEURS}

\section{GIAMPIERO SCAFOGLIO}

Université de Nice Sophia Antopolis, UMR 7264 CEPAM 